\title{
QUALIDADE DE ADESÃO DE JUNTAS COLADAS DE DIFERENTES ESPÉCIES COMERCIAIS DE MADEIRA
}

\author{
Alexandre Miguel do Nascimento ${ }^{*}$, Rosilei Aparecida Garcia ${ }^{1}$, Ricardo Marius Della Lucia ${ }^{2}$ \\ *Autor para correspondência: amn@ufrrj.br
}

\begin{abstract}
RESUMO: Neste estudo, objetivou-se determinar o efeito da densidade da madeira, do tipo de adesivo e da pressão de colagem na resistência ao cisalhamento de juntas coladas de quatorze espécies comerciais de madeira. As madeiras foram classificadas em três classes de densidade (Classe 1: menor que $0,55 \mathrm{~g} \mathrm{~cm}^{-3}$; Classe 2: entre 0,55 e 0,75 $\mathrm{g} \mathrm{cm}^{-3}$; e Classe $3:$ maior que $0,75 \mathrm{~g} \mathrm{~cm}^{-3}$ ) e as juntas foram coladas com dois adesivos: acetato de polivinila (PVA) e ureia-formaldeído (UF), sob duas diferentes pressões de colagem: 6 e $12 \mathrm{kgf} \mathrm{cm}^{-2}$. As juntas coladas com o adesivo PVA apresentaram maior resistência ao cisalhamento que aquelas coladas com o adesivo UF. Quanto à porcentagem de falha na madeira, o adesivo PVA também obteve o melhor desempenho, entretanto, somente as madeiras das Classes 1 e 2 atingiram os valores requeridos pela norma ASTM 3110. As espécies de madeira da Classe 3, coladas com UF, não atingiram os valores de resistência da madeira sólida. A pressão de colagem de $12 \mathrm{kgf} \mathrm{cm}^{-2}$ foi mais eficiente para as madeiras da Classe 3, tanto para a resistência ao cisalhamento quanto para a porcentagem de falha na madeira.
\end{abstract}

Palavras-chave: Densidade da madeira, polivinila acetato, pressão de colagem, resistência ao cisalhamento, ureia-formaldeído.

\section{ADHESION QUALITY OF GLUED JOINTS FROM DIFFERENT COMMERCIAL WOOD SPECIES}

ABSTRACT: The objective of this study was to determine the effect of wood density, adhesive type and gluing pressure on the shear strength of glued joints of fourteen commercial wood species. Wood pieces were classified in three density classes (Class 1: less than $0.55 \mathrm{~g} \mathrm{~cm}^{-3}$; Class 2: from 0.55 to $0.75 \mathrm{~g} \mathrm{~cm}^{-3}$; and Class 3: greater than $\left.0.75 \mathrm{~g} \mathrm{~cm}^{-3}\right)$ and joints bonded with two adhesives: polyvinyl acetate (PVA) and urea-formaldehyde (UF), under two different pressures: 6 and $12 \mathrm{kgf} \mathrm{cm}^{-2}$. Glued joints bonded with PVA adhesive presented higher shear strength than those bonded with UF adhesive. For percentage of wood failure, the PVA adhesive had the best performance, however, only Classes 1 and 2 reached the values required by ASTM 3110 standard. Glued joints from

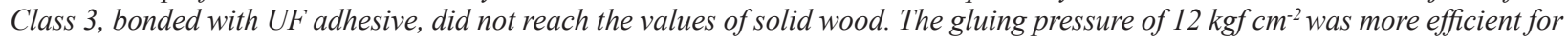
Class 3, for both shear strength and percentage of wood failure.

Key words: Wood density, polyvinyl acetate, gluing pressure, shear strength, urea-formaldehyde.

\section{INTRODUÇÃO}

Em 2008, a utilização da madeira serrada no Brasil, segundo a Associação Brasileira da Indústria de Madeira Processada Mecanicamente - ABIMCI (2008), atingiu a marca de 25,0 milhões $\mathrm{m}^{3}$, com predominância de madeira de folhosas, com 15,7 milhões $\mathrm{m}^{3}$ frente à produção de madeira de pinus com 9,3 milhões $\mathrm{m}^{3}$, representando um crescimento de $7 \%$ e 2,0\%, respectivamente, em relação a 2006. A madeira é um material construtivo e mobiliário e tem sido utilizada em níveis inferiores ao desejado, apesar da sua grande versatilidade. Mesmo considerando os aspectos favoráveis, tais como disponibilidade de um grande número de espécies, fácil trabalhabilidade e relação altamente interessante entre peso específico e resistência mecânica, o emprego de algumas madeiras torna-se, às vezes, difícil, em razão da falta de conhecimento sobre suas propriedades e seu desempenho em diferentes condições de serviço. Segundo
Grohmann (1998), é necessário o conhecimento preciso das características físicas e mecânicas da espécie a ser utilizada para que sua escolha e uso sejam satisfatórios à indústria ou à construção civil.

A adesão é um fator importante para a utilização dos recursos madeireiros e depende de fatores físicos, químicos e estruturais da madeira, os quais controlam a habilidade do adesivo de aderir às suas superfícies. Entre os fatores que afetam a qualidade de adesão da madeira, podemos citar a densidade, a porosidade, o teor de umidade, o teor de extrativos e a capacidade tampão (FOLLRICH et al., 2007). Segundo Forest Products Laboratory (2010), a resistência da linha adesiva aumenta com a densidade, até a faixa de 0,7 à $0,8 \mathrm{~g} \mathrm{~cm}^{-3}$. Para madeiras de densidades menores, a adesão é fácil e a resistência da madeira limita a resistência da linha adesiva, enquanto que, para madeiras de maiores densidades, torna-se difícil obter juntas de alta resistência ou com alta porcentagem de falha na madeira.

${ }^{1}$ Universidade Federal Rural do Rio de Janeiro - Seropédica, Rio de Janeiro, Brasil

${ }^{2}$ Universidade Federal de Viçosa - Viçosa, Minas Gerais, Brasil

Cerne, Lavras, v. 19, n. 4, p. 593-601, out./dez. 2013 
A adesão de madeiras de alta densidade é difícil, em razão da menor porosidade, ou seja, à maior espessura de parede e menor lume celular, limitando a penetração do adesivo à profundidade de uma ou duas células; e ao maior teor de extrativos, principalmente em madeiras tropicais, os quais afetam a molhabilidade da superfície da madeira e as características de fluxo, penetração e cura do adesivo (FOREST PRODUCTS SOCIETY, 2010).

Além das características da madeira, a qualidade de adesão também pode ser afetada pelo tipo de adesivo e outros fatores relacionados ao processo (tempo de cura, pressão de colagem, etc.). Carrasco (1989) relata que, para diferentes espécies de madeira, a pressão de colagem ideal varia entre 0,55 e $0,65 \mathrm{MPa}$ ou 5,6 e 6,6 $\mathrm{kgf} \mathrm{cm}^{-2}$, respectivamente. Nascimento et al. (2002) estudaram três níveis de pressão diferentes e observaram um aumento na resistência ao cisalhamento, para madeiras de Pinus e Corymbia citriodora, até a pressão $1,0 \mathrm{MPa}\left(10,2 \mathrm{kgf} \mathrm{cm}^{-2}\right)$, seguido de uma queda para pressões superiores. Segundo Forest Products Society (2010), para madeiras mais densas, uma maior pressão é requerida para aumentar a penetração do adesivo.

No Brasil, há predominância de madeiras com densidades mais elevadas e apesar do crescente número de pesquisas nessa área, poucos estudos têm abordado a qualidade da adesão das nossas madeiras, sejam nativas ou exóticas, com os adesivos existentes no mercado (SERPA et al., 2003; TIENNE et al., 2008; VITAL et al., 2006). Nesse contexto, neste estudo, objetivou-se determinar o efeito da densidade da madeira, do tipo de adesivo e da pressão de colagem na resistência ao cisalhamento de juntas coladas de quatorze espécies comerciais de madeira.

\section{MATERIAL E MÉTODOS}

\subsection{Material}

Quatorze espécies de madeiras comerciais foram utilizadas neste estudo tal como apresentado na Tabela 1. As amostras de madeira foram secas ao ar livre e, posteriormente, foram desdobradas, aplainadas e padronizadas na espessura em desengrossadeira, com sobremedida na largura e espessura. Após climatização, à $20^{\circ} \mathrm{C}$ e $65 \%$ de umidade relativa até peso constante, as dimensões das amostras foram ajustadas, seguindo a norma ASTM D 905 (AMERICAN SOCIETY FOR TESTING AND MATERIALS - ASTM, 1994c), com as seguintes dimensões finais: 19 x 50,8 x $304 \mathrm{~mm}$ (espessura $\mathrm{x}$ largura $\mathrm{x}$ comprimento). Posteriormente, as amostras foram coladas com dois tipos de adesivos: polivinila acetato (PVA) e ureia-formaldeído (UF), sob dois níveis

Tabela 1 - Lista das espécies estudadas (nome comum, nome científico e família).

Table 1 - List of studied species (common name, scientific name and family).

\begin{tabular}{lcc}
\hline Nome comum & Nome científico & Família $^{1}$ \\
\hline Angelim vermelho & Dinizia excelsa Ducke & Leguminosae-Mimosoideae \\
Angico vermelho & Piptadenia suaveolens Miq. & Leguminosae-Mimosoideae \\
Cedrorana & Cedrelinga catenaeformis Ducke & Leguminosae-Mimosoideae \\
Cedro rosa & Cedrela fissilis Vell. & Meliaceae \\
Cerejeira & Amburana cearensis (Fr. All.) A. C. Smith & Leguminosae-Papilionoideae \\
Cumaru & Dipteryx odorata (Aubl.) Willd. & Leguminosae-Papilionoideae \\
Garapa & Apuleia leiocarpa (Vog.) Macbr. & Leguminosae-Caesalpinioideae \\
Jatobá & Hymenaea courbaril L. & Leguminosae-Caesalpinioideae \\
Louro vermelho & Ocotea rubra Mez & Lauraceae \\
Pinus & Pinus sp. & Pinaceae \\
Parajú & Manilkara sp. & Sapotaceae \\
Tatajuba & Bagassa guianensis Aubl. & Moraceae \\
Tamboril & Enterolobium maximum Ducke & Leguminosae Mimosoideae \\
Vinhático & Plathymenia reticulata Benth. & Leguminosae Mimosoideae
\end{tabular}

${ }^{1}$ Fonte/Source: Fonseca et al. (2005).

Cerne, Lavras, v. 19, n. 4, p. 593-601, out./dez. 2013 
de pressão de colagem: 6 e $12 \mathrm{kgf} \mathrm{cm}^{-2}$, formando, assim, as juntas coladas. $\mathrm{O}$ adesivo PVA utilizado foi da Classe D-1, destinado a ambiente internos, para madeira abaixo de $15 \%$ de umidade. O teor de sólidos para os adesivos PVA (Cascorez) e UF (Cascamite) foram de 35,95 e 64,62\%, respectivamente. Para o adesivo UF, foi preparada uma mistura, em partes por peso, de resina (10), farinha de trigo como extensor (2), água (2) e catalisador (1). Para a resina UF, foi utilizado extensor para assemelhar à composição mais usual desse adesivo. Para ambos os adesivos, foram utilizados $200 \mathrm{~g}$ de sólidos por $\mathrm{m}^{2}$ de área colada, sendo as amostras prensadas em prensa hidráulica e testadas após 10 dias de climatização. As juntas coladas com UF, foram prensadas à temperatura de $60^{\circ} \mathrm{C}$.

Esse delineamento experimental resultou em 56 tratamentos com 2 juntas coladas de $38 \times 50,8 \times 304 \mathrm{~mm}$ (altura $\mathrm{x}$ largura $\mathrm{x}$ comprimento) para cada tratamento. De cada junta colada, foram obtidas 5 amostras, totalizando 10 repetições por tratamento.

\subsection{Ensaio de resistência ao cisalhamento na compressão paralela às fibras}

As amostras de madeira utilizadas para o ensaio de resistência ao cisalhamento na linha de cola foram dimensionadas e testadas de acordo com a norma ASTM D 905 (ASTM, 1994c). Após os ensaios de cisalhamento, os corpos-de-prova foram separados em duas partes, sendo a primeira destinada à determinação do teor de umidade e da densidade aparente, pelo método hidrostático, utilizandose o mercúrio no lugar da água; e a segunda parte destinada à medição da porcentagem de falha na madeira. A porcentagem de falha na madeira foi determinada de acordo com as prescrições da norma ASTM D 3110 (ASTM, 1994b), a qual exige um valor médio superior a $60 \%$ de falha na madeira e, pelo menos, $90 \%$ das amostras testadas com um mínimo de $30 \%$ de falha, sendo que nenhuma amostra pode apresentar $0 \%$ de falha.

Ainda, para efeito de comparação, a resistência ao cisalhamento da madeira sólida, para cada espécie, foi determinada pela norma ASTM D 143 (ASTM, 1994a), com 25 repetições. Posteriormente, foi calculada a resistência relativa, dada pela razão entre a média da resistência ao cisalhamento da junta colada e a média da resistência ao cisalhamento da madeira sólida.

\subsection{Análises estatísticas}

Os resultados obtidos para a resistência ao cisalhamento das juntas coladas foram submetidos à análise de variância (ANOVA). Comparações entre as médias, quando necessárias, foram feitas pelo teste de Tukey, ao nível de 5\% de significância. A variável porcentagem de falha na madeira, não teve distribuição normal e não foi possível sua normalização por meio de transformações, portanto, foi aplicado o teste não paramétrico de KruscallWallis, e comparado, posteriormente, à média dos postos dos tratamentos entre si, ao nível de 5\% de significância.

Após a obtenção dos resultados da densidade aparente, as quatorze espécies de madeiras utilizadas foram agrupadas em 3 classes de densidade: Classe 1: inferior à $0,55 \mathrm{~g} \mathrm{~cm}^{-3}$, Classe 2: entre 0,55 e $0,75 \mathrm{~g} \mathrm{~cm}^{-3}$ e Classe 3 : superior à $0,75 \mathrm{~g} \mathrm{~cm}^{-3}$. As espécies agrupadas na Classe 1 foram: vinhático, angelim, cedro rosa e cedrorana. As espécies agrupadas na Classe 2 foram: louro vermelho, pinus, tamboril, cerejeira e angico vermelho. As espécies agrupadas na Classe 3 foram: tatajuba, garapa, jatobá, cumaru e parajú. As análises estatísticas foram realizadas em função da classe de densidade aparente, do tipo de adesivo e da pressão de colagem.

\section{RESULTADOS E DISCUSSÃO}

Na Tabela 2, apresentam-se os resultados obtidos para a resistência ao cisalhamento na compressão paralela às fibras das juntas coladas com os adesivos PVA e UF e da madeira sólida, porcentagem de falha na madeira, densidade aparente das juntas coladas e da madeira sólida e resistência relativa para as quatorze espécies de madeira.

A densidade aparente média para a madeira sólida variou entre $0,34 \mathrm{e} 1,05 \mathrm{~g} \mathrm{~cm}^{-3}$ para as madeiras de vinhático e parajú, respectivamente, enquanto que a resistência média ao cisalhamento, oscilou entre 62 e $186,8 \mathrm{kgf} \mathrm{cm}^{-2}$, respectivamente, para vinhático e cumaru. Observa-se que a faixa de densidade aparente abrangeu dos mais baixos aos mais elevados valores encontrados para as madeiras brasileiras (Tabela 2).

Quando se compara a resistência ao cisalhamento das juntas coladas com PVA e UF, observa-se que todas as espécies estudadas apresentaram menores valores médios de resistência quando coladas com o adesivo UF, ou seja, este apresentou uma adesão mais fraca (Tabela 2).

Quanto à média da porcentagem de falha na madeira, para as juntas coladas com o adesivo PVA, somente as espécies das Classes 1 e 2 atingiram as exigências da norma ASTM D 3110 (ASTM, 1994b) (Tabela 2). No caso das juntas coladas com UF, apenas as espécies angelim vermelho e cedro rosa (Classe 1) atingiram as exigências da referida norma (Tabela 2).

Cerne, Lavras, v. 19, n. 4, p. 593-601, out./dez. 2013 
Tabela 2 - Médias obtidas para a resistência ao cisalhamento, porcentagem de falha na madeira, densidade aparente, teor de umidade de equilíbrio e resistência relativa para as quatorze espécies de madeira estudadas.

Table 2 - Shear strength means, percentage of wood failure, density, equilibrium moisture content and relative strength for fourteen wood studied species.

\begin{tabular}{|c|c|c|c|c|c|c|c|c|c|c|c|}
\hline \multirow{3}{*}{$\begin{array}{l}\text { Classe/ } \\
\text { Espécie }\end{array}$} & \multicolumn{6}{|c|}{ Linha de cola } & \multicolumn{3}{|c|}{ Madeira sólida } & \multirow{2}{*}{\multicolumn{2}{|c|}{$\begin{array}{c}\text { Resistência } \\
\text { relativa }\end{array}$}} \\
\hline & \multicolumn{2}{|c|}{$\mathrm{f}_{\mathrm{v} 0}\left(\mathrm{kgf} \mathrm{cm}^{-2}\right)$} & \multicolumn{2}{|c|}{ Falha (\%) } & \multicolumn{2}{|c|}{ DENS $\left(\mathrm{g} \mathrm{cm}^{-3}\right)$} & \multirow{2}{*}{$\begin{array}{c}\text { DENS } \\
\left(\mathrm{g} \mathrm{cm}^{-3}\right)\end{array}$} & \multirow{2}{*}{$\begin{array}{c}\mathrm{f}_{\mathrm{v} 0} \\
\left(\mathrm{kgf} \mathrm{cm}^{-2}\right)\end{array}$} & \multirow{2}{*}{$\begin{array}{l}\text { TUE } \\
(\%)\end{array}$} & & \\
\hline & PVA & UF & PVA & UF & PVA & UF & & & & PVA & UF \\
\hline \multicolumn{12}{|l|}{ Classe 1} \\
\hline Vinhático & 81,7 & 68,1 & $\underline{80,3}$ & 40,0 & 0,37 & 0,34 & 0,34 & 62,0 & 11,3 & 1,32 & 1,10 \\
\hline Angelim ver. & 111,2 & 84,2 & $\underline{98,5}$ & $\underline{72,9}$ & 0,52 & 0,44 & 0,44 & 76,5 & 11,5 & 1,45 & 1,10 \\
\hline Cedro rosa & 132,2 & 107,5 & $\underline{88,4}$ & $\underline{62,4}$ & 0,55 & 0,55 & 0,55 & 98,3 & 12,4 & 1,34 & 1,09 \\
\hline Cedrorana & 119,1 & 96,7 & $\underline{93,1}$ & 33,8 & 0,55 & 0,55 & 0,58 & 103,0 & 11,9 & 1,16 & $\underline{0,94}$ \\
\hline \multicolumn{12}{|l|}{ Classe 2} \\
\hline Louro ver. & 120,0 & 98,6 & $\underline{88,5}$ & 53,4 & 0,58 & 0,56 & 0,56 & 89,4 & 12,2 & 1,34 & 1,10 \\
\hline Pinus & 122,5 & 106,5 & $\underline{84,4}$ & 29,6 & 0,58 & 0,58 & 0,60 & 105,1 & 11,9 & 1,17 & 1,01 \\
\hline Tamboril & 140,4 & 121,2 & $\underline{91,0}$ & 49,8 & 0,57 & 0,59 & 0,57 & 100,3 & 10,4 & 1,40 & 1,21 \\
\hline Cerejeira & 139,3 & 113,3 & $\underline{79,9}$ & 44,1 & 0,67 & 0,67 & 0,65 & 112,6 & 10,4 & 1,24 & 1,01 \\
\hline Angico ver. & 175,9 & 143,1 & $\underline{72,0}$ & 18,8 & 0,73 & 0,70 & 0,65 & 133,6 & 11,8 & 1,32 & 1,07 \\
\hline \multicolumn{12}{|l|}{ Classe 3} \\
\hline Tatajuba & 189,9 & 147,1 & 42,1 & 16,8 & 0,89 & 0,86 & 0,84 & 156,5 & 11,5 & 1,21 & $\underline{0,94}$ \\
\hline Garapa & 172,6 & 135,0 & 23,1 & 11,1 & 0,89 & 0,89 & 0,88 & 160,5 & 11,4 & 1,08 & $\underline{0,84}$ \\
\hline Jatobá & 165,9 & 103,8 & 28,1 & 3,4 & 0,95 & 0,95 & 0,95 & 146,8 & 13,0 & 1,13 & $\underline{0,71}$ \\
\hline Cumaru & 190,4 & 86,2 & 13,5 & 2,5 & 1,02 & 1,02 & 1,04 & 186,8 & 10,6 & 1,02 & $\underline{0,46}$ \\
\hline Parajú & 212,9 & 178,3 & 31,3 & 32,4 & 1,02 & 1,02 & 1,05 & 185,1 & 11,8 & 1,15 & $\underline{0,96}$ \\
\hline
\end{tabular}

$\mathrm{f}_{\mathrm{v} 0}$ : resistência ao cisalhamento na compressão paralela às fibras. DENS: densidade aparente. TUE: teor de umidade de equilíbrio. Valores médios da porcentagem de falhas sublinhados são aqueles que atingiram o valor estabelecido pela norma ASTM 3110 (60\%). $f_{v 0}$ : shear strength in compression parallel to the fibers. DENS: density. TUE: equilibrium moisture content. Means values of percentage of wood failure highlighted are those that reached the values required by ASTM 3110 standard (60\%).

Quanto à distribuição de frequência da porcentagem de falhas, apenas o adesivo PVA, nas Classes 1 e 2, atingiu as exigências da norma, a qual estabelece que não pode haver nenhuma amostra com zero de porcentagem de falha e que ao menos $90 \%$ da amostras, apresente no mínimo $30 \%$ de falha (Figura 1).

Para a resistência relativa, quando usado o adesivo PVA, todos os índices foram superiores a 1 , indicando que a linha de cola foi mais resistente, em média, que a madeira sólida. O mesmo foi observado para as juntas coladas com o adesivo UF, com exceção da madeira de cedrorana (Classe 1) e todas as espécies da Classe 3, ou seja, madeiras de mais alta densidade aparente. Observou-se, também, que os índices de resistência relativa do adesivo PVA foram sempre superiores aos do adesivo UF (Tabela 2).

Cerne, Lavras, v. 19, n. 4, p. 593-601, out./dez. 2013



Figura 1 - Freqüência percentual da porcentagem de falha da madeira na linha de cola, em função do tipo de adesivo e classe de densidade.

Figure 1 - Percentage frequency of the percentage of wood failure in glued line, as a function of adhesive type and density class. 
Quanto ao teor de umidade de equilíbrio, o valor médio para todas as espécies de madeira foi de $11,6 \%$, sendo que o ambiente estava climatizado para $12 \%$ (Tabela 2). Nenhuma correlação significativa foi observada entre o teor de umidade de equilíbrio e a resistência ao cisalhamento e percentagem de falhas (Tabela 3).

$\mathrm{Na}$ Tabela 3, apresentam-se os coeficientes de correlação de Pearson entre as variáveis estudadas. Para as análises de correlação, foram consideradas a totalidade das amostras (incluindo os dois tipos de adesivos) e, posteriormente, as amostras coladas somente com um tipo de adesivo. Foram observadas correlações significativas entre: densidade aparente e resistência ao cisalhamento; e densidade aparente e porcentagem de falha na madeira. Os melhores coeficientes de correlação foram obtidos para o grupo de amostras coladas com o adesivo PVA. Para as análises de correlação entre a porcentagem de falha na madeira e a densidade aparente, para os dois adesivos utilizados, notou-se que a correlação para adesivo PVA foi maior que para o adesivo UF (Tabela 3 ).
Na Figura 2, apresenta-se a correlação existente entre a densidade aparente e a resistência ao cisalhamento das juntas coladas com PVA e UF, respectivamente. Os modelos estatísticos resultantes foram: $\mathrm{f}_{\mathrm{v} 0}=-20,689+$ 321,22dens $-107,181$ dens $^{2}$ para o adesivo PVA, sendo que os coeficientes estimadores dos parâmetros foram significativos e o coeficiente de determinação corrigido igual a 0,775 ; e $f_{v 0}=-28,459+350,073$ dens $^{-190,885 \text { dens }^{2}}$ para o adesivo UF com os coeficientes estimadores dos parâmetros significativos e o coeficiente de determinação corrigido igual a igual à 0,322 . Observou-se que a relação existente pode ser considerada quadrática para os dois tipos de adesivos. Na Classe 3, de densidade (maior que $0,75 \mathrm{~g} \mathrm{~cm}^{-3}$ ), quando usado UF, os valores de resistência tendem a ser tornar estáveis, alcançando o limite máximo de resistência e são, comparativamente, inferiores aos valores de resistência do PVA, os quais continuam aumentando quadraticamente em função dos valores de densidade. Isso prova que existe um substancial enfraquecimento da ligação colada com adesivo UF para madeira com densidade aparente superior à $0,75 \mathrm{~g} \mathrm{~cm}^{-3}$.

Tabela 3 - Coeficientes de correlação de Pearson entre as variáveis dependentes estudadas.

Table 3 - Pearson's correlation between all studied dependent variables.

\begin{tabular}{|c|c|c|c|c|}
\hline \multicolumn{5}{|c|}{ Coeficiente de correlação de todas as observações } \\
\hline & $\begin{array}{c}\text { Resistência ao } \\
\text { cisalhamento }\end{array}$ & $\begin{array}{c}\text { Percentagem } \\
\text { de falha }\end{array}$ & TUE & DENS \\
\hline Resistência ao cisalhamento & 1,00 & $-0,13$ & $-0,02$ & $\underline{0,64}^{* *}$ \\
\hline Percentagem de falha & & 1,00 & $-0,08$ & $\underline{-0,54^{* *}}$ \\
\hline TUE & & & 1,00 & 0,04 \\
\hline \multicolumn{5}{|c|}{ Coeficiente de correlação das amostras coladas com PVA } \\
\hline & $\begin{array}{c}\text { Resistência ao } \\
\text { cisalhamento }\end{array}$ & $\begin{array}{c}\text { Percentagem } \\
\text { de falha }\end{array}$ & TUE & DENS \\
\hline Resistência ao cisalhamento & 1,00 & $\underline{-0,57} * *$ & 0,02 & $\underline{0,87} * *$ \\
\hline Percentagem de falha & & 1,00 & $-0,02$ & $\underline{-0,71}^{* *}$ \\
\hline TUE & & & 1,00 & 0,06 \\
\hline \multicolumn{5}{|c|}{ Coeficiente de correlação das amostras coladas com UF } \\
\hline & $\begin{array}{c}\text { Resistência ao } \\
\text { cisalhamento }\end{array}$ & $\begin{array}{c}\text { Percentagem } \\
\text { de falha }\end{array}$ & TUE & DENS \\
\hline Resistência ao cisalhamento & 1,00 & $-0,10$ & 0,05 & $\underline{0,50}^{* *}$ \\
\hline Percentagem de falha & & 1,00 & $-0,04$ & $\underline{-0,50} * *$ \\
\hline TUE & & & 1,00 & 0,02 \\
\hline
\end{tabular}

TUE: teor de umidade de equilíbrio. DENS: densidade aparente. ** Significativo à $99 \%$ de probabilidade.

TUE: equilibrium moisture content. DENS: density. ** Significant at the 0.01 probability level.

Cerne, Lavras, v. 19, n. 4, p. 593-601, out./dez. 2013 

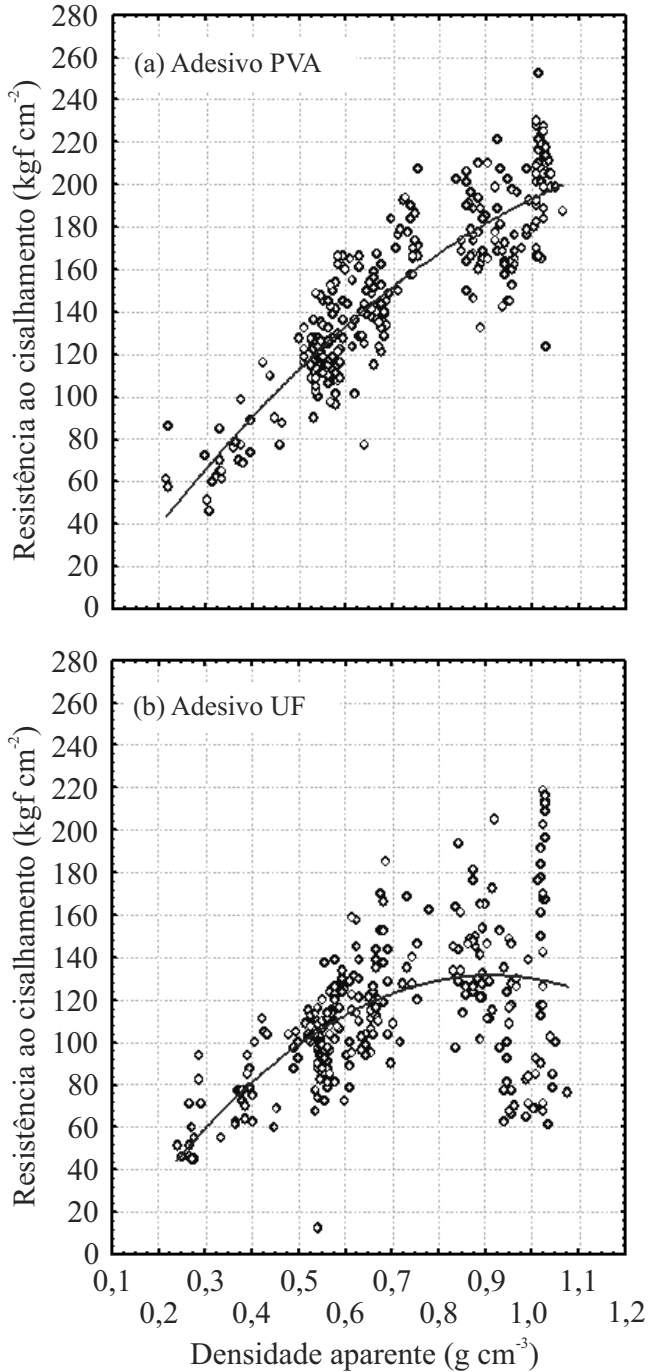

Figura 2 - Correlação entre a resistência ao cisalhamento e a densidade aparente das juntas coladas com os adesivos PVA e UF, respectivamente.

Figure 2 - Correlation between shear strength and density of glued joints bonded with PVA and UF adhesives, respectively.

Na Tabela 4, a correlação entre a porcentagem de falha e a densidade aparente foi categorizada em função do tipo de adesivo e da classe de densidade. Foram observadas correlações significativas para a Classe 2 de densidade, para ambos os adesivos (PVA e UF) e para a Classe 1 do adesivo PVA. Para a Classe 3, não foram encontradas correlações significativas para nenhum dos dois adesivos. Observa-se que, para Classe 1 de densidade, o valor de correlação significativo, foi positivo, enquanto que na Classe 2 de densidade, os valores de correlações significativos foram negativos, revelando que a porcentagem de falha diminui a medida que as densidades médias oscilam entre $0,55 \mathrm{e}$ $0,75 \mathrm{~g} \mathrm{~cm}^{-3}$, independentemente do adesivo utilizado. A porcentagem de falha está associada a maior penetração do adesivo nas camadas superficiais da madeira. Quando se cola madeira de mais alta densidade, as quais possuem menor porosidade, há uma menor disponibilidade de vazios para a penetração do adesivo, dificultando o processo de adesão. Isso explica a ausência de correlação entre a porcentagem de falha na madeira e densidades superiores à $0,8 \mathrm{~g} \mathrm{~cm}^{-3}$.

Tabela 4 - Correlação entre a porcentagem de falha na madeira e as classes de densidade para os adesivos PVA e UF.

Table 4 - Correlation between percentage of wood failure and density classes for PVA (A) and UF (B) adhesives, respectively.

\begin{tabular}{lccc}
\hline Adesivo & Classe 1 & Classe 2 & Classe 3 \\
\hline PVA & $\underline{0,2614^{*}}$ & $\underline{-0,3367^{*}}$ & $-0,1838$ \\
UF & 0,0190 & $\underline{-0,3927^{*}}$ & 0,0860 \\
Dois adesivos & 0,160 & $\underline{-0,250^{*}}$ & $-0,040$ \\
\hline
\end{tabular}

** Significativo à $95 \%$ de probabilidade.

** Significant at the 0.05 probability level.

Na Figura 3a, apresenta-se a comparação entre as médias de resistência ao cisalhamento das juntas coladas em função da classe de densidade para os adesivos PVA e UF. O adesivo PVA apresentou um melhor desempenho que o adesivo UF, principalmente para a Classe 3 de densidade. O melhor desempenho do adesivo PVA em relação ao UF é que este ultimo foi colado na temperatura de $60^{\circ} \mathrm{C}$, abaixo dos valores usados para produção de chapas laminadas ou aglomeradas. Outra possível razão, para tal desempenho, foi à utilização do extensor, farinha de trigo, que causa um enfraquecimento da linha adesiva. Suzuki et al. (1986 citado por RIVER, 1994), testando formulações de adesivo UF, obteve menor valor de resistência para a formulação que fez uso de farinha de trigo como extensor.

Na Tabela 5, apresenta-se a análise de variância (ANOVA) para a resistência ao cisalhamento das juntas coladas em função da classe de densidade, do tipo de adesivo e da pressão de colagem. Observou-se um efeito significativo para a classe de densidade, o tipo de adesivo e as interações duplas (Classe*Adesivo e Classe*Pressão de colagem).

Cerne, Lavras, v. 19, n. 4, p. 593-601, out./dez. 2013 

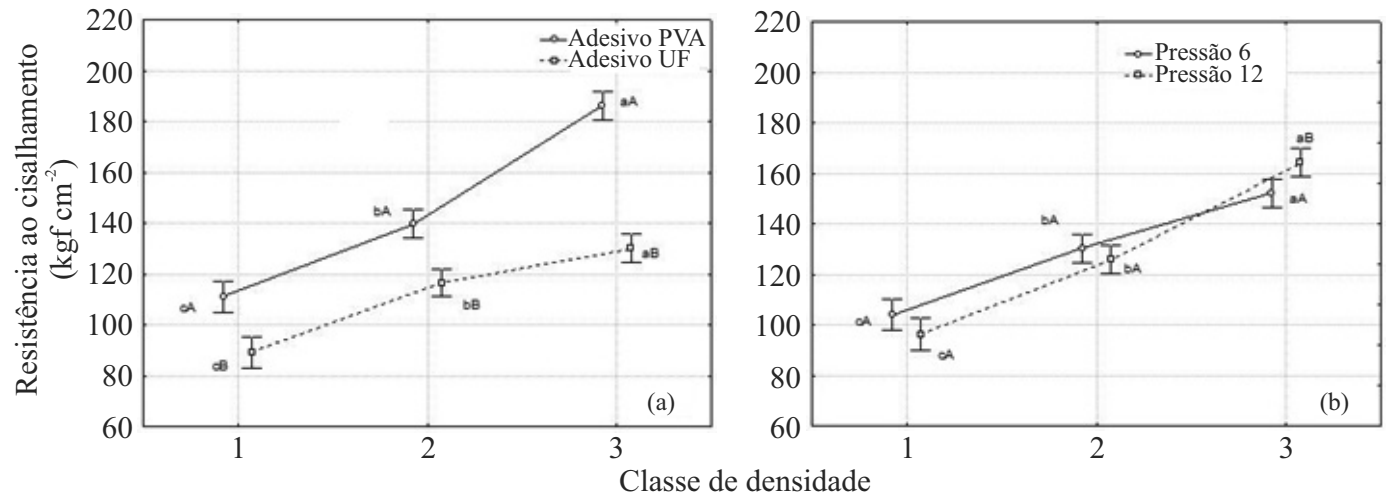

Figura 3 - Interação entre classe de densidade e tipo de adesivo (a) e classe de densidade e pressão de colagem (b) para resistência ao cisalhamento. Médias com a mesma letra não são estatisticamente diferentes pelo teste de Tukey à $5 \%$ de probabilidade. Letras minúsculas: comparação entre as médias de diferentes classes de densidade para o mesmo adesivo ou mesma pressão de colagem. Letras maiúsculas: comparação entre médias de diferentes adesivos ou diferentes pressões de colagem.

Figure 3 - Interaction between density class and adhesive type (a) and density class and gluing pressure (b) for shear strength. Means with the same letter are not significantly different by Tukey test at $0.05 \%$ probability level. Lower case letters: comparison between means of the different density classes for the same adhesive or gluing pressure. Capital letters: comparison between means of the different adhesives or different gluing pressures.

Tabela 5 - Resultados obtidos para a análise de variância (ANOVA) da resistência ao cisalhamento das juntas coladas.

Table 5 - Analysis of variance (ANOVA) for shear strength of the glued joints.

\begin{tabular}{lccccc}
\hline Fonte de variação & SQ & GL & QM & F & P $<$ \\
\hline Classe & $3,02 \mathrm{E}+05$ & 2 & $1,51 \mathrm{E}+05$ & $187,2^{* *}$ & 0,000 \\
Adesivo & $1,58 \mathrm{E}+05$ & 1 & $1,58 \mathrm{E}+05$ & $195,2^{* *}$ & 0,000 \\
Pressão de colagem & 3 & 1 & 3 & $0,0^{\text {ns }}$ & 0,9540 \\
\hline Interação & & & & \\
\hline Classe*Adesivo & $3,65 \mathrm{E}+04$ & 2 & $1,83 \mathrm{E}+004$ & $22,6^{* *}$ & 0,000 \\
Classe*Pressão de colagem & $1,07 \mathrm{E}+04$ & 2 & 5342 & $6,6^{* *}$ & 0,001 \\
Adesivo*Pressão de colagem & 422 & 1 & 422 & $0,5^{\mathrm{ns}}$ & 0,4700 \\
Classe*Adesivo*Pressão de colagem & 1303 & 2 & 652 & $0,8^{\mathrm{ns}}$ & 0,4470 \\
\hline
\end{tabular}

** Significativo à $99 \%$ de probabilidade. ${ }^{\text {ns }}$ Não significativo.

** Significant at the 0.01 probability level. ${ }^{n s}$ Not significant.

A resistência ao cisalhamento foi maior para as classes de densidade superiores, algo já esperado, visto que, a resistência está diretamente relacionada à densidade, pois paredes celulares espessas são capazes de suportar maiores tensões (FOREST PRODUCTS LABORATORY, 2010).

Na Figura 3b, apresenta-se a comparação entre as médias de resistência ao cisalhamento das juntas coladas em função da classe de densidade para as diferentes pressões de colagem (6 e $12 \mathrm{kgf} \mathrm{cm}^{-2}$ ). Não foi encontrada diferença significativa entre as pressões de colagem utilizadas, nas classes de densidades inferiores, entretanto, para a Classe 3 de densidade, as juntas produzidas com uma pressão de 12 $\mathrm{kgf} \mathrm{cm}{ }^{-2}$ apresentaram um melhor desempenho.

$\mathrm{Na}$ Figura 4, apresenta-se a porcentagem de falha na madeira em função da classe de densidade para os diferentes tipos de adesivos e pressões de colagem. O adesivo PVA teve um desempenho superior ao adesivo UF para todas as classes de densidade (Figura 4a). As Classes 1 e 2 apresentaram os melhores valores de porcentagem de falha para os dois adesivos utilizados, entretanto, somente as juntas coladas com o adesivo PVA nas Classes 1 e 2 atingiram as exigências da norma ASTM 3110 (ASTM, 1994b).

Cerne, Lavras, v. 19, n. 4, p. 593-601, out./dez. 2013 

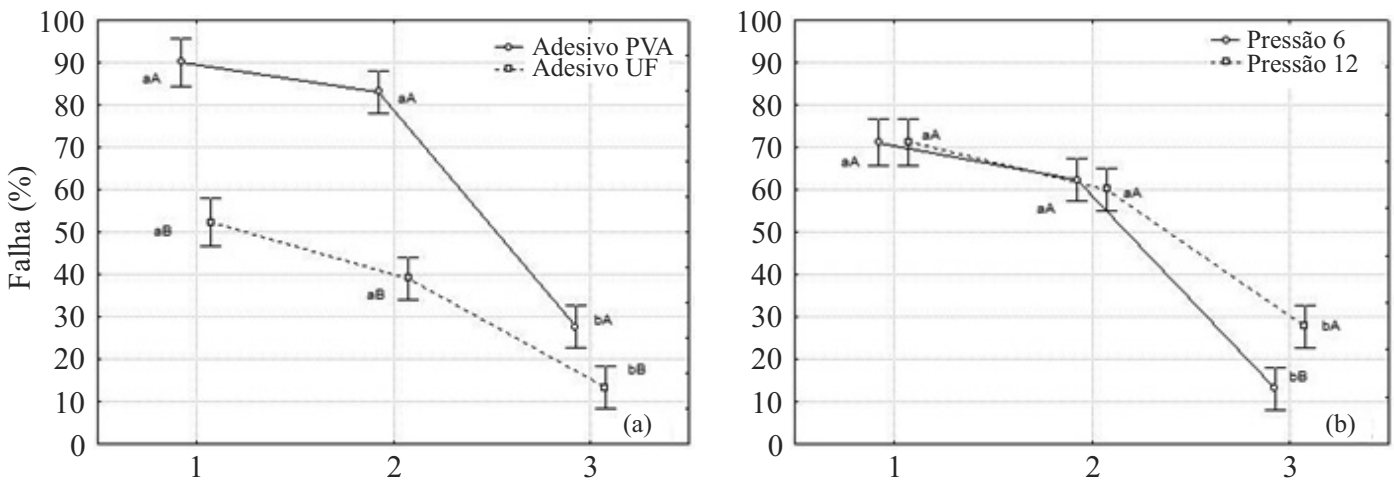

Figura 4 - Interação entre classe de densidade e tipo de adesivo (a) e classe de densidade e pressão de colagem (b) para porcentagem de falha na madeira. Médias com a mesma letra não são estatisticamente diferentes pelo teste de comparações múltiplas entre as médias dos pontos à 5\% de probabilidade. Letras minúsculas: comparação entre as médias de diferentes classes de densidade para o mesmo adesivo ou mesma pressão de colagem. Letras maiúsculas: comparação entre médias de diferentes adesivos ou diferentes pressões de colagem.

Figure 4-Interaction between density class and adhesive type (a) and density class and gluing pressure (b) for percentage of wood failure. Means with the same letter are not significantly different by multiple comparison test at $0.05 \%$ probability level. Lower case letters: comparison between means of the different density classes for the same adhesive or gluing pressure. Capital letters: comparison between means of the different adhesives or different gluing pressures.

Na Figura 4b, apresenta-se a porcentagem de falha na madeira em função da pressão de colagem e da classe de densidade. $\mathrm{O}$ efeito da pressão de colagem foi significativo somente na maior classe de densidade (Classe 3 ), na qual as juntas prensadas à $12 \mathrm{kgf} \mathrm{cm}^{-2}$ apresentaram os melhores resultados.

Segundo Forest Products Laboratory (2010), altos valores de falha na madeira são encontradas para espécies de madeira com densidade aparente abaixo de $0,75 \mathrm{~g} \mathrm{~cm}^{-3}$ à $12 \%$ de umidade. Acima desse valor, a percentagem de falha diminui. Isso ocorre, em razão da menor porosidade da madeira e da presença de lumens de menor diâmetro, o que dificulta a penetração e o ancoramento mecânico do adesivo à madeira, até duas camadas de células a partir da superfície.

Maiores pressões de colagem são necessárias para uma devida compressão de madeira mais densas e, consequentemente, mais rígidas. Tais pressões promovem melhor contato entre a superfície da madeira e o adesivo (FOREST PRODUCTS LABORATORY, 2010). Nascimento et al. (2002) encontraram o dobro da percentagem de falhas, para madeira de Pinus sp., usando adesivo resorcinol, quando a pressão de colagem foi alterada de 0,6 MPa para 1,0 MPa. Para madeira de Eucalyptus citriodora a percentagem de falha aumentou em $35 \%$, quando se alterou a pressão de colagem de $0,6 \mathrm{MPa}$ para 1,4 MPa. Amadeira de Eucalyptus citriodora apresentou uma densidade aparente de $1,05 \mathrm{~g} . \mathrm{cm}^{-3}$ a $12 \%$ de umidade, enquanto que a madeira Pinus apresentou 0,54 g. $\mathrm{cm}^{-3} \mathrm{de}$ densidade nas mesmas condições de umidade.

Sugere-se que novos trabalhos sejam feitos, avaliando a penetração de adesivos em madeiras densas, especialmente aos adesivos relativos a este estudo, com ênfase ao PVA e suas variações.

\section{CONCLUSÕES}

Para todas as espécies de madeira estudadas, o adesivo PVA apresentou valores de resistência ao cisalhamento superiores aos obtidos pelo adesivo UF.

Para todas as classes de densidade, o adesivo PVA teve um desempenho superior à UF quanto à porcentagem de falha na madeira, entretanto, somente as madeiras das Classes 1 e 2 atingiram os valores requeridos pela norma ASTM 3110.

Espécies de madeira da Classe de densidade 3, coladas com UF, não atingiram os valores de resistência da madeira sólida.

A maior pressão de colagem foi mais eficiente para as madeiras da Classe 3, tanto para a resistência ao cisalhamento quanto para a porcentagem de falha na madeira.

Cerne, Lavras, v. 19, n. 4, p. 593-601, out./dez. 2013 


\section{REFERÊNCIAS}

AMERICAN SOCIETY FOR TESTING AND MATERIALS. Standard methods of testing small clear specimens of timber: ASTM D 143. Annual Book of A.S.T.M. Standards, Philadelphia, v. 4, v. 10, p. 24-65, 1994 a.

AMERICAN SOCIETY FOR TESTING AND MATERIALS Standard specification for adhesives used in nonstructural glued lumber products: ASTM D 3110. Annual Book of A.S.T.M. Standards, Philadelphia, v. 15, v. 6, p. 184-192, 1994b.

AMERICAN SOCIETY FOR TESTING AND MATERIALS. Standard test method for strength properties of adhesive bonds in shear by compression loading: ASTM D 905. Annual Book of A.S.T.M. Standards, Philadelphia, v. 15, v. 6, p. 20-26, 1994c.

\section{ASSOCIAÇÃO BRASILEIRA DE MADEIRA} PROCESSADA MECANICAMENTE. Estudo setorial 2008. São Paulo, 2008. 56 p.

CARRASCO, E. V. M. Resistência, elasticidade e distribuição de tensões nas vigas retas de madeira laminada colada. 1989. $120 \mathrm{f}$. Tese (Doutorado em Engenharia de Estruturas) - Escola de Engenharia de São Carlos, São Carlos, 1989.

FOLLRICH, J.; TEISCHINGER, A.; GINDL, W.; MÜLLER, $\mathrm{U}$. Effect of grain angle on shear strength of glued end grain to flat grain joints of defect-free softwood timber. Wood Science and Technology, New York, v. 41, p. 501-509, 2007.

FONSECA, C. N.; LISBOA, P. L. B.; URBINATI, C. V. A xiloteca (Coleção Walter A. Egler) do Museu Paraense Emílio
Goeldi. Boletim do Museu Paraense Emílio Goeldi, Série Ciências Naturais, Belém, v. 1, n. 1, p. 65-140, 2005.

FOREST PRODUCTS LABORATORY. Wood handbook: wood as an engineering material: general technical report FPL-GTR-190. Madison: USDA, 2010. 508 p.

GROHMANN, S. Z. Vigas de madeira laminada colada com lâminas pré-classificadas de Eucalyptus grandis. 1998. 120 p. Dissertação (Mestrado em Engenharia Civil) Universidade Federal de Santa Catarina, Florianópolis, 1998.

NASCIMENTO, A. M.; LELIS, R. C. C.; COSTA, D. L.; OLIVEIRA, C. S. Comportamento de ligações adesivas em madeira de reflorestamento. Floresta e Ambiente, Seropédica, v. 9, n. 1, p. 54-62, 2002.

RIVER, B. H. Fracture of adhesive-bonded wood joints. In: PIZZI, A.; MITTAL, K. L. (Ed.). Handbook of adhesive technology. New York: M. Dekker, 1994. p. 151-177.

SERPA, P. N.; VITAL, B. R.; DELLA LUCIA, R. M.; PIMENTA, A. S. Avaliação de algumas propriedades da madeira de Eucalyptus grandis, Eucalyptus saligna e Pinus elliottii.

Revista Árvore, Viçosa, v. 27, n. 5, p. 723-733, set./out. 2003.

TIENNE, D. L. C.; NASCIMENTO, A. M.; GARCIA, R. A.; SILVA, D. B. Adhesion quality of quaruba cedar wood glued joints under internal and external service conditions. Floresta e Ambiente, Seropédica, v. 15, n. 1, p. 20-33, 2008.

VITAL, B. R.; MACIEL, A. S.; DELLA LUCIA, R. M. Qualidade de juntas coladas com lâminas de madeira oriundas de três regiões do tronco de Eucalyptus grandis, Eucalyptus saligna e Pinus elliottii. Revista Árvore, Viçosa, v. 30, n. 4, p. 637-644, jul./ago. 2006.

Recebido: 14 de julho de 2011; aceito: 24 de maio de 2013. 
